This item was submitted to Loughborough's Research Repository by the author.

Items in Figshare are protected by copyright, with all rights reserved, unless otherwise indicated.

\title{
Analytical characterization of damping in gear teeth dynamics under hydrodynamic conditions
}

PLEASE CITE THE PUBLISHED VERSION

http://dx.doi.org/10.1016/j.mechmachtheory.2015.08.007

PUBLISHER

(C) Elsevier

VERSION

AM (Accepted Manuscript)

\section{PUBLISHER STATEMENT}

This work is made available according to the conditions of the Creative Commons Attribution-NonCommercialNoDerivatives 4.0 International (CC BY-NC-ND 4.0) licence. Full details of this licence are available at: https://creativecommons.org/licenses/by-nc-nd/4.0/

\section{LICENCE}

CC BY-NC-ND 4.0

\section{REPOSITORY RECORD}

Liu, F.H., Stephanos Theodossiades, L.A. Bergman, A.F. Vakakis, and D.M. McFarland. 2019. "Analytical Characterization of Damping in Gear Teeth Dynamics Under Hydrodynamic Conditions". figshare. https://hdl.handle.net/2134/19062. 


\title{
Analytical Characterization of Damping in Gear Teeth Dynamics Under Hydrodynamic Conditions
}

\author{
F.H. Liu ${ }^{1}$, S. Theodossiades ${ }^{2}$, L. Bergman ${ }^{3}$, A. Vakakis ${ }^{4}$ and D.M. McFarland ${ }^{3}$
}

\author{
1. Department of Automotive Engineering \\ Yancheng Institute of Technology, Yancheng, 224051, P. R. China \\ 2. Wolfson School of Mechanical and Manufacturing Engineering \\ Loughborough University, Loughborough, LE11 3TU, UK \\ Tel: +44 1509 227664, Fax: +44 1509 227648, e-mail: S.Theodossiades@lboro.ac.uk \\ 3. Department of Aerospace Engineering \\ University of Illinois at Urbana-Champaign, Urbana, IL 61801, USA \\ 4. Department of Mechanical Science and Engineering \\ University of Illinois at Urbana-Champaign, Urbana, IL 61801, USA
}

\begin{abstract}
Using an analytical method we characterize damping and stiffness in lightly loaded, lubricated gear pairs at different operating speeds and lubricant temperatures. This is accomplished by employing the trace method to approximate and model the hysteresis loop of the lubricant reaction, thus recording the energy transformation mechanism during the gear teeth oscillatory motion. The method can be expanded for use in a variety of problems where hydrodynamic vibro-impacts lead to energy dissipation.
\end{abstract}

\section{Introduction and governing equations}

Lubricant damping properties play an important role in the dynamics of gear pairs. For lightly loaded operating conditions, the critical viscous damping ratio, $\zeta$, can be generally estimated as being inversely proportional to the gear teeth meshing stiffness:

$$
\zeta=\frac{c}{2 \sqrt{k_{m} m_{e}}}
$$


where $c$ is the viscous lubricant damping coefficient during gear mesh, $k_{m}$ is the average teeth mesh stiffness and $m_{e}$ is the gear pair's equivalent mass. It is understood that the damping ratio implicitly depends on the operating conditions (rotational speed, load and temperature), which influence the rigidity of the lubricant film developed between the teeth (Guilbault et al., 2012; Li and Kahraman, 2011; Amabili and Rivola, 1997).

The aim of this work is to present an analytical method for calculating the variation of the damping ratio in gear teeth contacts under lightly loaded hydrodynamic conditions, reflecting any lubricant effects. To this end, we consider standard involute helical gear teeth geometry without modifications, and we model the gear pair as a lumped parameter torsional system, considering rotational displacements $\left(\theta_{p}, \theta_{g}\right)$ only (Fig. 1). The subscripts $p$ and $g$ refer to the pinion and gear, respectively. The lubricant film can physically act on the drive $\left(h_{d}\right)$ and coast side $\left(h_{c}\right)$ of the teeth contacts, respectively. Under light loads, it is reasonable to assume that the centres of both gears are not moving axially or laterally (the vibrations of individual shafts in transmissions play no essential role in the system dynamics, Dimarogonas et al., 2013) and that the teeth flanks are rigid.

The motion of the pinion can be a priori described by

$$
\dot{\theta}_{p}(t)=\dot{\vartheta}_{m}+\sum_{i=1}^{\infty} \dot{\vartheta}_{f}^{i} \sin \left(i \omega t+\varphi_{i}\right)
$$

In the above equation, $\dot{\vartheta}_{m}$ is the mean value of the pinion's rotational speed; $\omega$ is the excitation frequency (which - in the case of automotive applications for example - depends on the engine type, number of cylinders and crankshaft configuration); $\dot{\vartheta}_{f}^{i}$ and $\varphi_{i}$ are the fluctuation amplitudes of the rotational speed and the phase of the $i^{\text {th }}$ order, respectively.

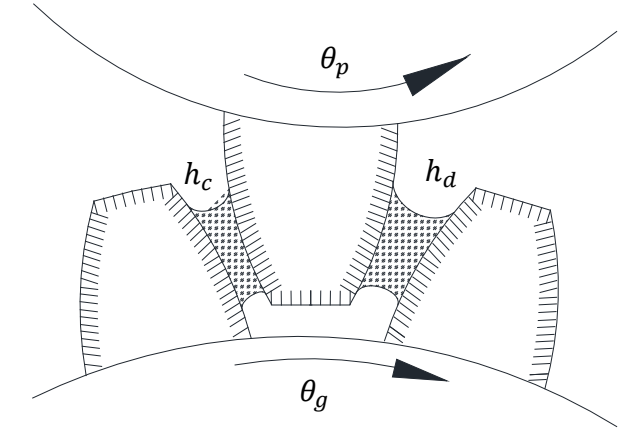

Fig. 1. The physical problem and model used.

The corresponding equation of motion for the gear wheel is given in its general form as 


$$
I_{g} \ddot{\theta}_{g}-T_{g}^{w}\left(\theta_{g}, \theta_{p}\right)+T_{g}^{f}\left(\theta_{g}, \theta_{p}\right)=-T^{p}\left(\theta_{p}, \theta_{s}\right)
$$

where $I_{g}$ is the gear mass moment of inertia and $\theta_{s}$ is the output shaft rotation angle. The torque generated by the lubricant reaction $\left(T_{g}^{w}\right)$, hydrodynamic flank friction $\left(T_{g}^{f}\right)$ and hydrodynamic Petrov friction $\left(T^{p}\right.$ ) have been described in detail in Theodossiades et al. (2007). Only the elements needed for the gear teeth meshing lubricant damping calculation will concern us in this work. Through analytical solution of the Reynolds's equation, the lubricant reaction force $\left(F^{w}\right)$ between the teeth flanks was derived as follows for the iso-viscous rigid prevailing lubrication conditions in the case studied (Theodossiades et al., 2007, Rahnejat, 1985),

$$
F^{w}=\frac{2 L \eta R_{e q} u_{e}}{h_{d} h_{c}}\left[2 C_{b}-\left(h_{c} \lambda_{d}+h_{d} \lambda_{c}\right)\right]
$$

where $L$ is the gear face width; $\eta$ is the dynamic viscosity of the lubricant; $R_{e q}$ is the equivalent radius of curvature normal to the line of contact; $u_{e}$ is the speed of the oil film entraining motion; and $2 C_{b}$ is the total gear backlash, whilst $h_{d}$ and $h_{c}$ correspond to the lubricant film thickness at the drive and coast sides, as shown in Fig. 1. The squeeze-to-roll ratios $\left(\lambda_{d}, \lambda_{c}\right)$ signify the contributions of the squeeze action of the lubricant at the drive and coast sides, respectively, as (Theodossiades et al., 2007, Rahnejat, 1985),

$$
\lambda_{j}=\left\{\begin{array}{cc}
\frac{3 \pi}{2 u_{e} \sqrt{2 h_{j} / R_{e q}}} \frac{\partial h_{j}}{\partial t} & \frac{\partial h_{j}}{\partial t}<0 \\
0 & \frac{\partial h_{j}}{\partial t} \geq 0
\end{array} \quad j=c, d\right.
$$

The friction introduced by the roller bearings, upon which the gear wheel is mounted on the retaining shaft, can be calculated as (Shigley and Mischke, 1989),

$$
F^{p}=\frac{2 \pi \eta v l R_{s}}{C_{s}}
$$

where $R_{s}$ is the radius of the output shaft, $C_{s}$ and $l$ are the clearance and length of the conformal contact surfaces between the shaft and the idle gear, and $v$ is the entraining velocity of the lubricant. 


\section{Analytical damping characterization}

The problem of describing the energy transformation mechanism in a pair of oscillating gears under hydrodynamic lubrication conditions can reduce to that of identifying two functions (involving the angular displacement and speed of each gear wheel as free variables); one function represents the contribution from the nonlinear stiffness of the lubricant and the other from its viscous damping capabilities, so that their amalgamation eventually forms the classical hysteresis loop mechanism. The trace method considers the hysteresis loop as the summation of a non-linear, non-hysteretic function related to the cycle shape and an equivalent viscous damping evaluated from the area enclosed by one complete cycle of oscillations. According to the trace method, the hysteresis loop represented in the displacement-force plane can be approximated by an ellipse of the same amplitude, enclosing the same area (Badrakhan, 1977). The above approach allows us to divide the contact teeth mesh force $\left(F^{w}\right)$ into two parts, originating from the stiffness and damping actions (Lankarani and Nikravesh, 1990)

$$
F_{W}=F_{k}+F_{c}
$$

where the first term represents the elastic force, and the second term accounts for the energy dissipation during the entire period of motion. This model assumes that energy loss is due to the oil damping of the system, dissipated mostly in the form of heat (affecting the gear pair's efficiency). Assuming that the response is a narrow-band process, the gear teeth dynamic transmission error function $x$ (the motion along the line of teeth contact) takes the form during steady-state oscillations of

$$
x=x_{a} \sin (\varphi)
$$

where $\varphi=\omega t+\phi$ is the phase of the displacement with respect to the excitation force and $x_{a}$ is the amplitude of the dynamic transmission error.

Since backlash is present in all geared systems (either by design and/or due to manufacturing errors and wear), rather than using a polynomial or bilinear function (Badrakhan, 1977, Badrakhan, 1987), the trace representing the non-hysteretic function can be approximated by a piecewise linear curve. The trace force $\left(F_{k}\right)$ represented in the force-displacement plot by the "trace" is defined as 


$$
F_{k}(x)=\left\{\begin{array}{cc}
k_{2} x-\left(k_{2}-k_{1}\right) C_{o} & x>C_{o} \\
k_{1} x & -C_{o} \leq x \leq C_{o} \\
k_{2} x+\left(k_{2}-k_{1}\right) C_{o} & x<-C_{o}
\end{array}\right.
$$

where $k_{1}$ and $k_{2}$ correspond to the two linear stiffness regions of the lubricant hydrodynamic force, as shown in Fig. 2. The transition is assumed to take place at $\pm C_{o}$ with the location of those limits depending on the loop shape. Note that the latter is expected to change dramatically when any of the following parameters changes: Excitation frequency, gear rotation speed and lubricant temperature. Once the shape of the hysteresis loop corresponding to this force model is known (through the modelled system dynamics or experimental data), the coefficients $k_{1}$ and $k_{2}$ can be obtained as

$$
k_{1}=\frac{F_{k}\left(C_{o}\right)}{C_{o}}, k_{2}=\frac{F_{k}\left(x_{a}\right)-F_{k}\left(C_{o}\right)}{x_{a}-C_{o}}
$$

By implementing equation (2.2) in (2.3) and setting $\sin \left(\varphi_{e}\right)=C_{o} / x_{a}$, equation (2.3) becomes

$$
F_{k}(\varphi)=\left\{\begin{array}{cc}
k_{2} x_{a} \sin (\varphi)-\left(k_{2}-k_{1}\right) C_{o} & \sin (\varphi)>\sin \left(\varphi_{e}\right) \\
k_{1} x_{a} \sin (\varphi) & \sin \left(-\varphi_{e}\right) \leq \sin (\varphi) \leq \sin \left(\varphi_{e}\right) \\
k_{2} x_{a} \sin (\varphi)+\left(k_{2}-k_{1}\right) C_{o} & \sin (\varphi)<\sin \left(-\varphi_{e}\right)
\end{array}\right.
$$

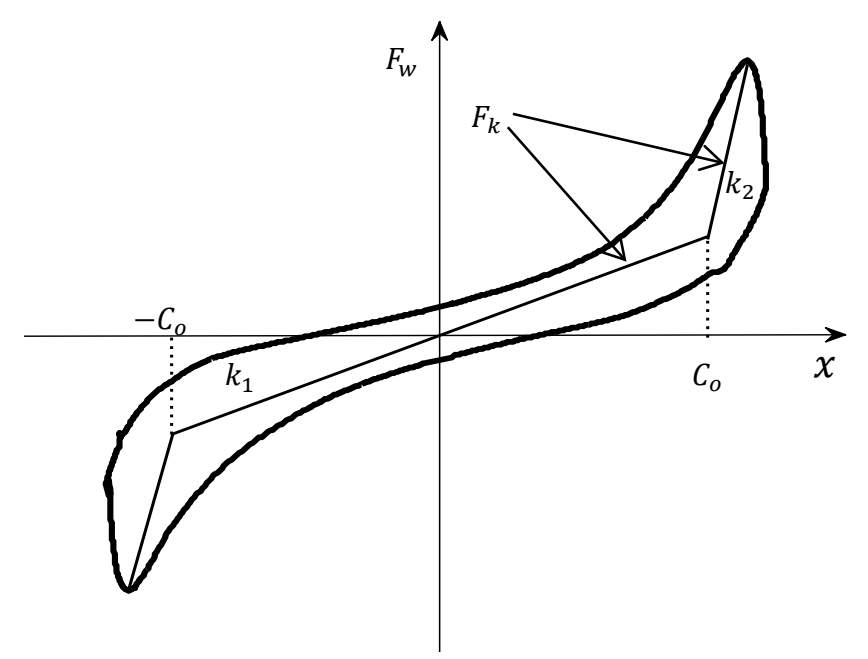

Fig. 2. Nonlinear teeth contact force $F_{w}$ and trace force function $F_{k}$ (hysteresis loop)

The limits in the above piecewise linear function can be written as

$$
\begin{aligned}
& \sin (\varphi)>\sin \left(\varphi_{e}\right) \Leftrightarrow \varphi_{e} \leq \varphi \leq \pi-\varphi_{e} \\
& \sin \left(-\varphi_{e}\right) \leq \sin (\varphi) \leq \sin \left(\varphi_{e}\right) \Leftrightarrow 2 \pi-\varphi_{e} \leq \varphi \leq 2 \pi+\varphi_{e}, \pi-\varphi_{e} \leq \varphi \leq \pi+\varphi_{e} \\
& \sin (\varphi)<\sin \left(-\varphi_{e}\right) \Leftrightarrow \pi+\varphi_{e} \leq \varphi \leq 2 \pi-\varphi_{e}
\end{aligned}
$$


Therefore, the trace force function becomes

$$
F_{k}(\varphi)=\left\{\begin{array}{cc}
k_{2} x_{a} \sin (\varphi)-\left(k_{2}-k_{1}\right) C_{o} & \varphi_{e} \leq \varphi \leq \pi-\varphi_{e} \\
k_{1} x_{a} \sin (\varphi) & 2 \pi-\varphi_{e} \leq \varphi \leq 2 \pi+\varphi_{e} \\
& \pi-\varphi_{e} \leq \varphi \leq \pi+\varphi_{e} \\
k_{2} x_{a} \sin (\varphi)+\left(k_{2}-k_{1}\right) C_{o} & \pi+\varphi_{e} \leq \varphi \leq 2 \pi-\varphi_{e}
\end{array}\right.
$$

Then, the maximum stored energy in the lubricant can be found analytically by integration as

$$
\begin{aligned}
E & =\oint F_{k}(x) d x=x_{a} \oint F_{k}(\varphi) \cos (\varphi) d \varphi \\
& =x_{a}^{2}\left(\left(2-\sin \left(\varphi_{e}\right)\right) \sin \left(\varphi_{e}\right) k_{1}+\frac{1}{2}\left(3-\cos \left(2 \varphi_{e}\right)-4 \sin \left(\varphi_{e}\right)\right) k_{2}\right)
\end{aligned}
$$

The damping force can be written as

$$
F_{c}=c \dot{x}=c \omega x_{a} \cos (\varphi)
$$

The energy lost per cycle due to the action of an equivalent viscous damper is evaluated by integrating the damping force through the hysteresis loop, assuming that its characteristics during the lubricant compression and restitution phases are the same (Bert, 1973), and is given by

$$
\Delta E=\oint F_{c} d x=c \omega x_{a}^{2} \int_{0}^{2 \pi} \cos ^{2}(\varphi) d \varphi=\pi c \omega x_{a}^{2}
$$

The following equation is used for determining the damping ratio when experimentally obtained hysteresis loop data are available (Dareing and Johnson, 1975; Rao and Yap, 1995):

$$
\zeta \approx \frac{\sigma}{2}=\frac{1}{4 \pi} \frac{\Delta E}{E}=\frac{c \omega}{4\left(2-\sin \left(\varphi_{e}\right)\right) \sin \left(\varphi_{e}\right) k_{1}+2\left(3-\cos \left(2 \varphi_{e}\right)-4 \sin \left(\varphi_{e}\right)\right) k_{2}}
$$

where the loss coefficient $\sigma$ is defined as the ratio of the energy dissipated per radian divided by the maximum strain energy. Although the viscous damping depends on the physical parameters of the gear system (including the lubricant properties, teeth geometry, teeth sliding speed as well as the teeth meshing force), the damping ratio is directly proportional to the excitation frequency $(\omega)$ and inversely proportional to the stiffness coefficients $k_{1,2}$. 


\section{Lubricant stiffness and damping characteristics}

The excitation is assumed to be harmonic (as in the case of automotive applications with transmission gear pairs driven by an internal combustion engine), comprising a single term $(j=1)$ with the pinion nominal speed superimposed upon engine order vibrations (with amplitude at $2 \%$ of the nominal speed amplitude). These conditions correspond to drivetrains equipped with a modern palliative for vibration reduction (dual-mass flywheel). The lubricant properties correspond to those of synthetic gear oil (indicative value of dynamic viscosity $\eta_{20^{\circ} \mathrm{C}}=0.1154 \mathrm{~Pa} \cdot \mathrm{s}$ ).

According to Fig. 1, the two virtual springs (gear meshing teeth on the drive and coast sides) act in parallel. Each one corresponds to the equivalent stiffness of two springs in series (one from the solid teeth and one from the lubricant), where the spring with the lower stiffness governs the contact (this being the stiffness of the lubricant for hydrodynamic conditions). The choice of the right spring mechanism determines the interactions between the teeth flanks, which behave as nonlinear springs following the meshing cycle variation; their stiffness coefficients are related to the film thickness and teeth contact kinematics. The lubricant stiffness is calculated by differentiating equation (1.4) with respect to the film thickness $\left(h_{d}\right.$ and $\left.h_{c}\right)$, and is

$$
K_{l u b}=\underbrace{\left|\frac{\partial F_{w}^{a}}{\partial h_{d}}\right|}_{K_{d}}+\underbrace{\left|\frac{\partial F_{w}^{c}}{\partial h_{c}}\right|}_{K_{c}}
$$

The lubricant stiffness on the driving and coast sides is given by:

for $\partial h_{j} / \partial t<0 \quad(j=d, c)$

$$
K_{j}=\frac{L \eta R_{e q}}{4 h_{j}^{3}}\left(9 \sqrt{2} \pi R_{e q} \sqrt{\frac{h_{j}}{R_{e q}}} \frac{\partial h_{j}}{\partial t}-8 h_{j} u_{e}\right)
$$

and for $\partial h_{j} / \partial t \geq 0$

$$
K_{j}=-\frac{2 L \eta R_{e q} u_{e}}{h_{j}^{2}}
$$

Fig. 3 exhibits the time history of the lubricant stiffness at $60^{\circ} \mathrm{C}$. It can be seen that the stiffness values when teeth contact is established on the coast side are slightly higher than those when the contact occurs on the drive side (as a result of the severe double-sided oscillations occurring in this temperature). Note that $t_{f}=2 \pi / \omega$ corresponds to one period of the motion (due to the external excitation). Fig. 4 shows the combined and individual (per teeth pair) stiffness along the line of 
action for the case of pinion constant velocity over three consecutive gear teeth meshing cycles $\left(t_{c}=\varphi_{m p} / \dot{\vartheta}_{m}\right.$, corresponding to the teeth meshing period with $\varphi_{m p}$ being the meshing cycle gear rotation angle). The contact loss is clearly a source of non-linearity, which is apparently unavoidable in gear systems. Although there has been only a $2 \%$ perturbation of the pinion speed (in the case of Fig. 3), the stiffness variation shows a very different behaviour compared to the results of Fig. 4.

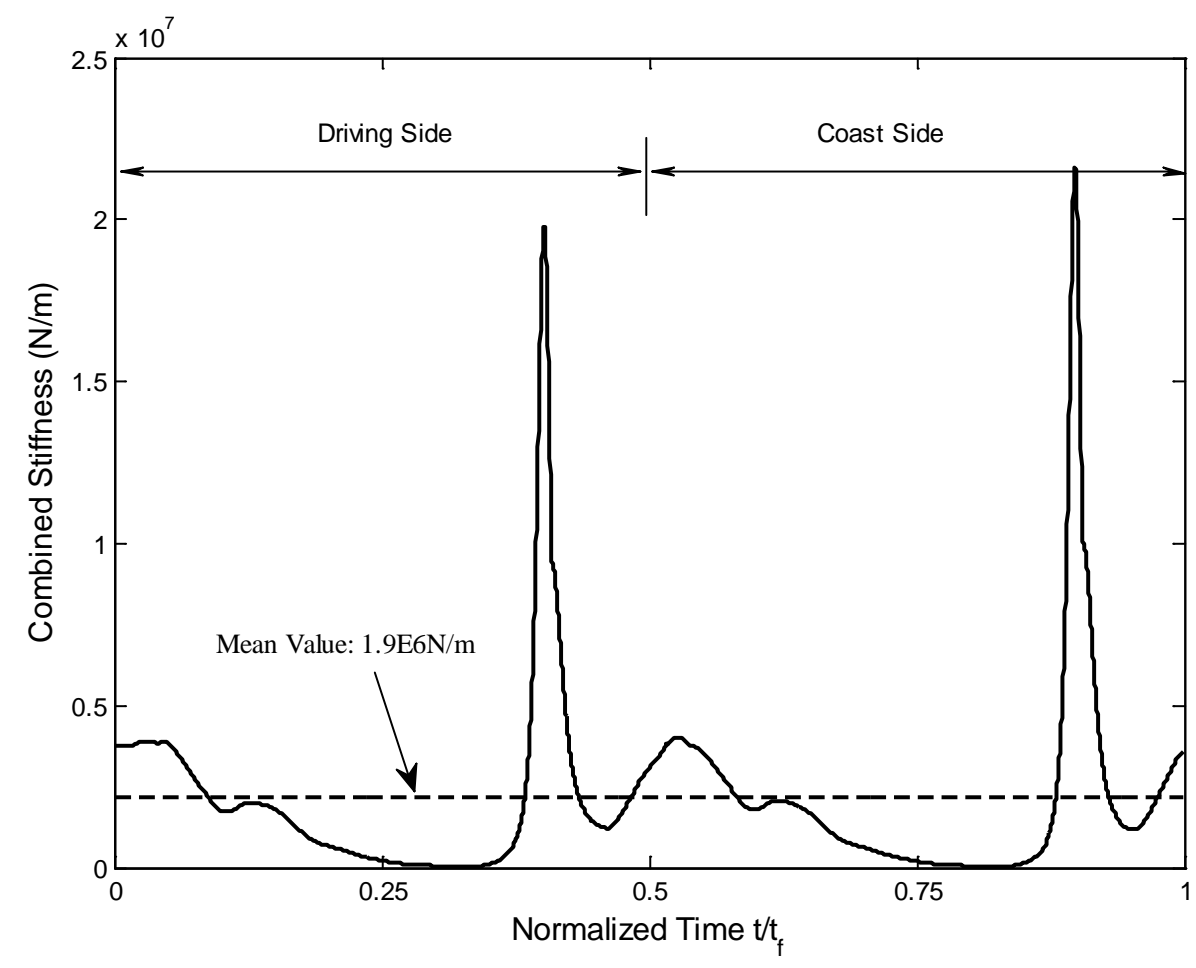

Fig. 3. Mesh stiffness variation at $60^{\circ} \mathrm{C}$ :

— : total stiffness of pairs; ----: mean value of the total stiffness. 


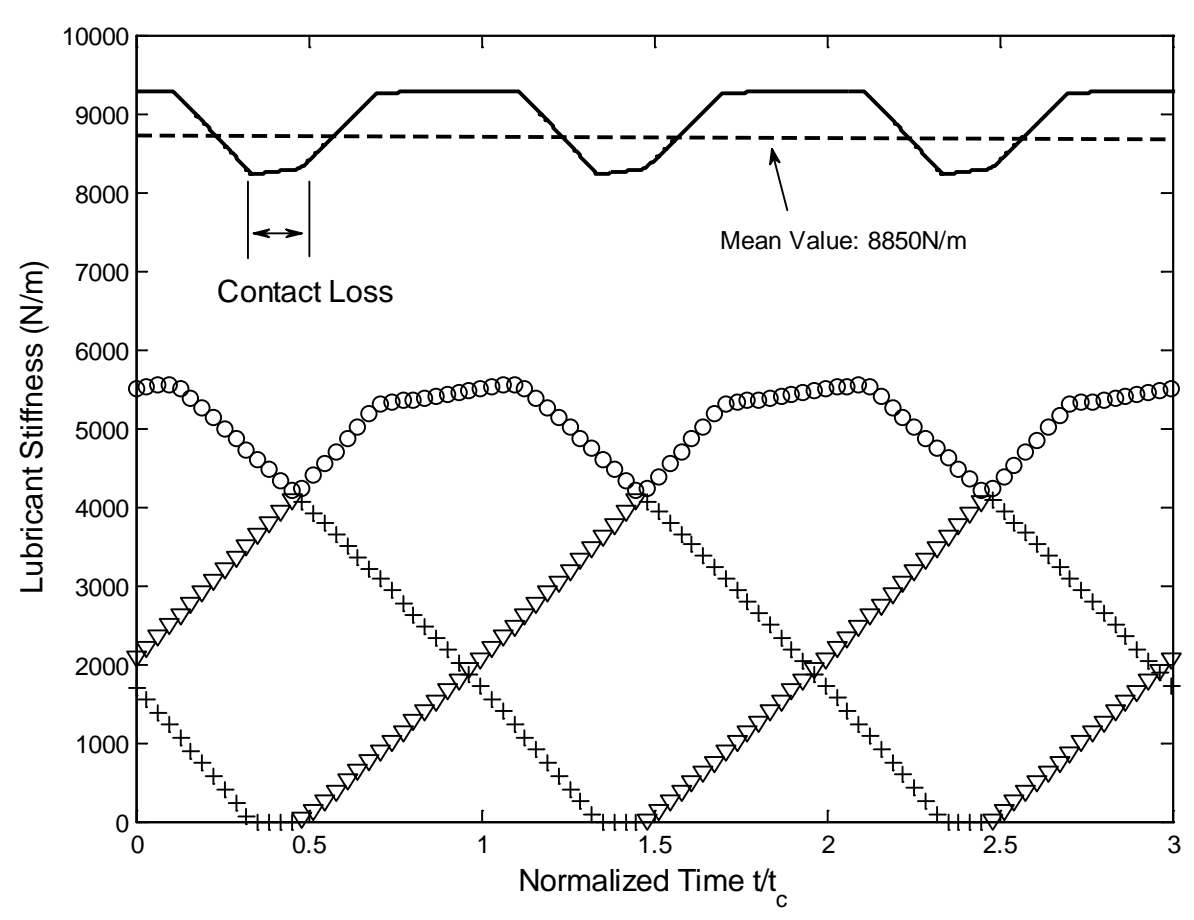

Fig. 4. Mesh stiffness at $60^{\circ} \mathrm{C}$ for the case of constant pinion speed $\left(\dot{\theta}_{p}=800 \mathrm{RPM}\right)$. $\nabla \nabla \nabla$ Stiffness of teeth pair 1; ooo Stiffness of teeth pair 2; $\times \times \times$ Stiffness of teeth pair 3;

—: total stiffness of pairs; ----: mean value of the total stiffness.

During teeth contact, the damping behaviour is mainly controlled by the oil squeeze action; parameters such as the lubricant viscosity (and thus, temperature), as well as the speed of entraining motion play a significant role. Fig. 5 shows the shape of the hysteresis loop at different oil temperatures. While the temperature increases, the loop shape changes dramatically with the second region stiffness coefficient $\left(k_{2}\right)$ increasing sharply. At low temperatures the motion of the gear wheel is nearly harmonic (following the gear meshing frequency). When the temperature increases, the lubricant's inability to support the load induces higher harmonics in the motion (distorting the shape of the hysteresis loop, as it can be seen in Fig. 5). 




Fig. 5. Hysteresis loop of the lubricant load capacity. $-20^{\circ} \mathrm{C} ; \cdots .40^{\circ} \mathrm{C} ;---60^{\circ} \mathrm{C}$

Depending on the pinion operating speed (and the severity of any perturbation effects), the minimum oil film thickness decreases as the oil temperature increases. This behaviour has been described by Larsson (1997) (squeeze effect mechanism). To better illustrate this relation, Fig. 6 depicts the damping ratio variation at different temperatures and for three different pinion speeds. Generally, it can be seen that for a given temperature, higher operation speed increases the damping ratio (lubricant viscous damping capacity). The increase of damping ratio occurring in parallel to the decrease of the minimum film thickness leads to augmentation of the dissipative energy; the latter is due to higher perturbations and severe double-sided teeth vibro-impacts (drive - coast drive teeth contacts). 


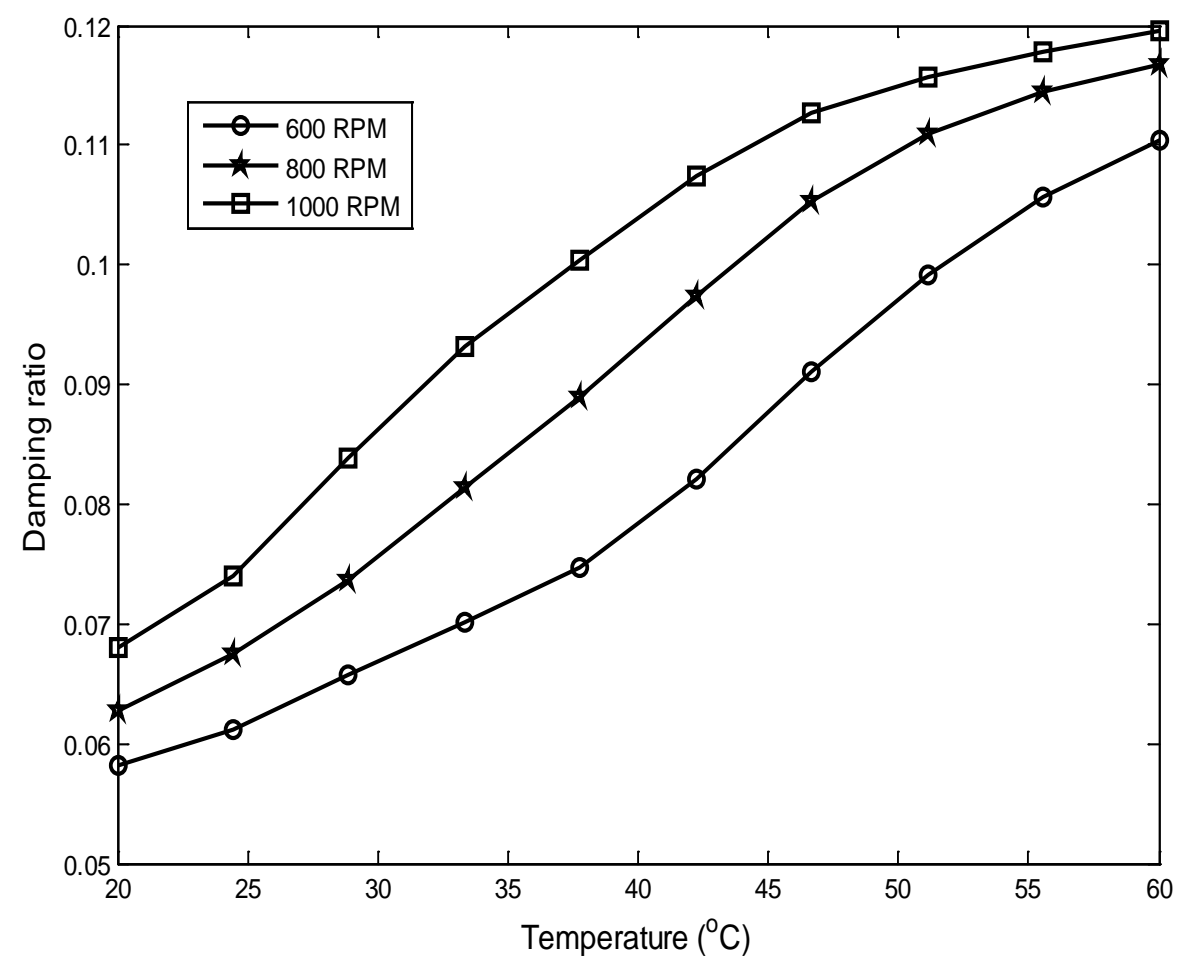

Fig. 6. Damping ratio variation with respect to pinion speed and oil temperature

\section{Concluding remarks}

We have provided a method that predicts the damping content and describes the energy exchange mechanism in gear teeth lubricated contacts under hydrodynamic conditions. The trace method has been employed, establishing a piecewise linear relationship between the gear teeth dynamic transmission error and the hydrodynamic contact force in the teeth oscillatory response hysteresis loop. The method can be employed to quantify the amount of energy dissipated in the teeth contacts of gear pairs that are engaged without transferring significant loads (the majority of gear pairs in modern transmissions) and support the analysis from an energy efficiency perspective. In addition, the method presented could be applied in the design process of systems with collaborating shafts (rotordynamics) under varying temperature and lubricant properties in order to determine their natural frequencies (to avoid resonance). The aim is to reduce the number of unknown parameters, which is usually an order of magnitude higher than the available design data, thus eliminating uncertainty further. 


\section{Acknowledgements}

Dr F. Liu would like to acknowledge the financial support from the National Natural Science Foundation of China (Grant No.51305378), the Jiangsu Provincial Key Laboratory of Automotive Engineering (QC201306) and the Research Laboratory of Mechanical Vibration (MVRLAB).

\section{References}

Guilbault, R., S. Lalonde, and M. Thomas. (2012). Nonlinear damping calculation in cylindrical gear dynamic modeling. Journal of Sound And Vibration.

Li, S. and A. Kahraman. (2011). A spur gear mesh interface damping model based on elastohydrodynamic contact behaviour. International Journal of Powertrains, 1(1), 4-21.

Amabili, M. and A. Rivola. (1997). Dynamic analysis of spur gear pairs: steady-state response and stability of the sdof model with time-varying meshing damping. Mechanical Systems and Signal Processing, 11(3), 375-390.

Dimarogonas, A.D., Paipetis, S.A. and Chondros, T.G. (2013). Analytical Methods in Rotor Dynamics, Elsevier.

Theodossiades, S., O. Tangasawi, and H. Rahnejat. (2007). Gear teeth impacts in hydrodynamic conjunctions promoting idle gear rattle. Journal of Sound And Vibration, 303(3-5), 632-658.

Rahnejat, H. (1985). Computational modelling of problems in contact dynamics. Engineering Analysis, 2(4), 192-197.

Shigley, J.E. and Mischke, C.R. (1989). Mechanical Engineering Design (Mcgraw-Hill Series in Mechanical Engineering). Mcgraw-Hill College.

Badrakhan, F. (1977). Etude approchee d'un systeme a hysteresis. International Journal of Non-linear Mechanics, 12(1), 1-7.

Lankarani, H. and P. Nikravesh. (1990). A contact force model with hysteresis damping for impact analysis of multibody systems. Journal of Mechanical Design, 112, 369.

Badrakhan, F. (1987). Rational study of hysteretic systems under stationary random excitation. International Journal of Non-linear Mechanics, 22(4), 315-325.

Bert, C.W. (1973). Material damping: An introductory review of mathematic measures and experimental technique. Journal of Sound And Vibration, 29(2), 129-153.

Dareing, D. and K. Johnson. (1975). Fluid film damping of rolling contact vibrations. Journal of Mechanical Engineering Science, 17(4), 214-218.

Rao, S.S. and Yap, F.F. (1995). Mechanical vibrations. Addison-Wesley New York.

Larsson, R. (1997). Transient non-Newtonian elastohydrodynamic lubrication analysis of an involute spur gear. Wear, 207(1-2), 67-73. 